\title{
Supporting Information \\ Minimal Surfaces on unconcatenated polymer rings in melt
}

\author{
Jan Smrek* and Alexander Y. Grosberg \\ Center for Soft Matter Research and Department of Physics, \\ New York University, New York, NY 10003, USA
}

\section{CONTENTS}

I. Surface minimization procedures
A. Surface preparation
B. Surface minimization

II. Minimal surface on space-filling curve

III. Freely fluctuating unknotted ring conformations 3

IV. Fraction of minimal surface area close to the boundary

V. Ring threading example

VI. Fraction of symmetric penetrations

VII. Threading length distribution

VIII. Separation length distribution

IX. Relative ring-ring mean square displacement: statistics details

References

7

\section{SURFACE MINIMIZATION PROCEDURES}

\section{A. Surface preparation}

Initially, the surface spanned on the ring of $N$ monomers consists of $N$ triangles, two vertices of which are defined by two subsequent monomers of the ring and the third vertex lies in the center of mass of the ring. These triangles are twice refined into smaller ones by subdividing each triangle into four smaller ones ending up with $16 N$ triangles. All triangle edges and vertices that are part of the bond segments of the ring are held fixed and form the boundary of the surface. Care is taken to perform equiangulation and vertex averaging when needed to have a well behaved mesh.

* js5013@nyu.edu

\section{B. Surface minimization}

1 As mentioned in the main text the evolution of the sur1 face by surface tension can lead to numerical problems 1 as the boundary can support minimal surfaces of different topology. For example a boundary with the shape close to doubly folded circle is known to support also a minimal surface with topology of the Möbius band [1-3]. The numerical surface tension flow (also known as mean curvature flow - see below) can drive the surface towards the different topology class which is observed to happen through a localized triangle thinning that requires progressively shorter time step and frequent mesh corrections through equiangulations or vertex averaging (see

4 [4] for details), to behave well numerically. This however can lead to an effective stalled configuration even before 5 the surface is minimal. We observed such effect also for a few of the rings in the melt, because locally some segments of the rings may resemble the doubly folded circle and the surface develops similar thin twisted neck [2]. However all we need is to get "sufficiently close" to the minimum area surface of disk topology. To do so, the well behaved regions of the surface must have enough time to relax and therefore we employed two different strategies.
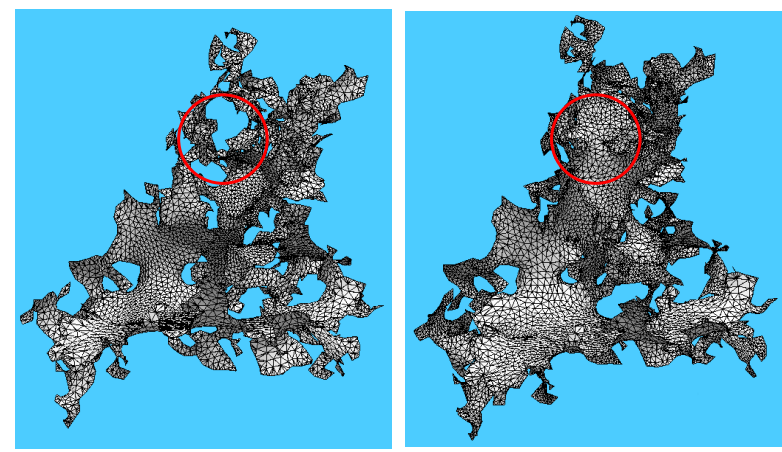

FIG. S1. Example of a minimal surface ring with $N=1600$ obtained from two different protocols. Left column: flow with zero temperature, Right column: "violent" annealing protocol that ended with a long run at a low temperature. The red circles mark the localized rearrangement differences. In this instance the surface obtained from the flow protocol was of smaller area (by about $7 \%$ - the largest value from the ensemble)

Firstly, we systematically weed out skinny triangles by means of deleting their edges and merging them with their neighbors. Such weeding of triangles can cause in some cases change of topology [4], but we checked by 
computing the Euler characteristic that the disk topology did not change during the evolution for our rings. The final surfaces have still more than $10 N$ triangles. The evolution was stopped when the surface area was not changing for more than $0.2 \%$ for a long time. In some cases the evolution was nevertheless stopped because of the topology restriction and decreasing time step. To ensure we are close to the minimal surface of disk topology we introduced a small temperature by means of displacing each vertex by a small (less than 0.1 of the minimal edge length) random vector at each iteration step and run further evolution. This prevents the evolution to fall in the singularity as that is compensated by the random displacements. This approach ensures that the surface is minimal on large scales and the singularity is localized at a few vertices as shown below. Even after long run we observed only insignificant area differences due to more (or less) efficient triangulations of the surface.

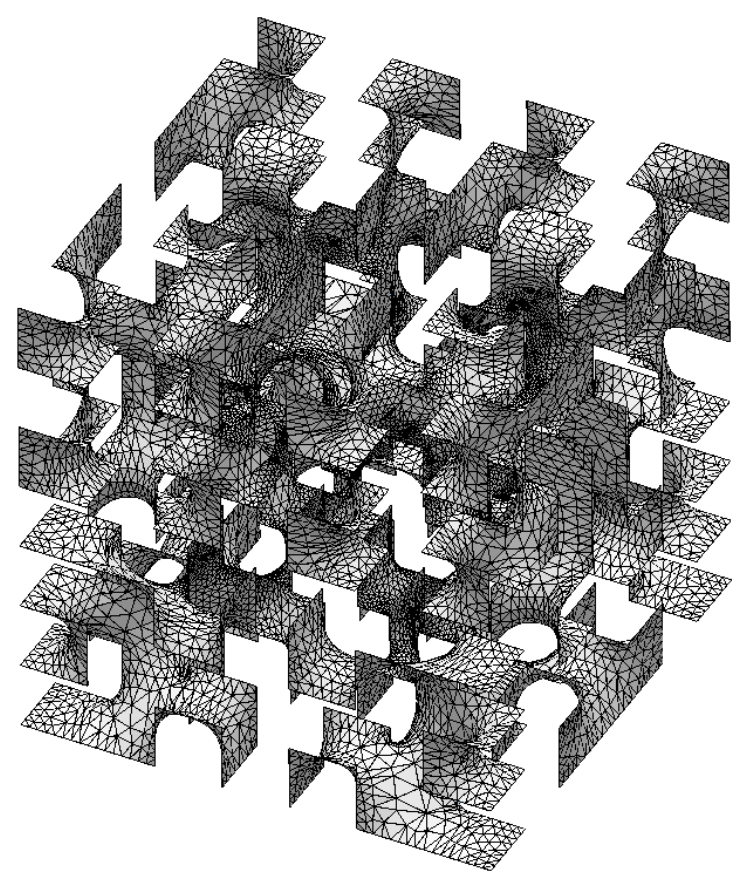

FIG. S2. Minimal surface for the Moore curve of $N=512$, reminiscent of the periodic Scherk surface [5].

Secondly, to avoid the singularity trap and triangle weeding we used also another minimization protocol that also allowed to check the importance of competing local minima of the surface area. In this "violent" protocol, the initial refinements were followed by a long period of large kicks that were about 20 times greater than the initial edge lengths to erase the bias of the initial configuration. Then the minimization continued at the low temperature with equiangulations as before. The minimization was finished if the area was stable (changes less than $0.2 \%$ ) for a long time. We observed that these conformations containing all the $16 N$ triangles (but naturally different triangulations) are mostly the same as ones obtained with the first faster protocol. In some cases we observe small local surface differences such as the one in Figure S1, reflected in a different value of the minimal area. Importantly, the area distribution was the same for both protocols. This allows us to claim that the scaling of the area with $N$ in the case of rings from the melt is not a consequence of a properly chosen protocol, but a generic property of the ring conformation ensemble.

We also checked that our surfaces are minimal and the singularity, if any, is localized to a few vertices by calculating a discreet approximation of a mean curvature at every free vertex as in [4]. Note that as the pressure on both sides of the ring's surface is the same the surface tension force due to Young-Laplace pressure is proportional to the local mean curvature of the surface. Therefore also the minimizing condition on the surface area for a given boundary, is equivalent to the condition that mean curvature of the surface is zero at every point. Inverse local mean curvature is a length scale $R_{c}$ characterizing a difference from a locally flat (i.e. minimal) surface. If $R_{c} \gg R_{g}$ for all vertices the surface is locally very close to mean zero curvature (hence minimal). Indeed this is the case for most of the vertices $\left(R_{c}>8 R_{g}\right.$ for $99 \%$ of all the vertices for $N=1600$ rings). The few outliers have on average $R_{c}$ not smaller than $R / 10 \simeq 1$ in monomer size units. Nevertheless the measure indicates that the surface is minimal to a reasonable extent except maybe at a few points that might indicate the locations of the singularities.

Overall the singularities do not play very important role in our case for the following reason. Their effect is more pronounced for larger rings as the more crumpled conformations of the rings are present allowing for possibly more singularities. Thus finding a true minimal surfaces of disk topology can only decrease the exponent of area scaling. However the area scaling with $N$ can not be weaker than linear, that we have found.

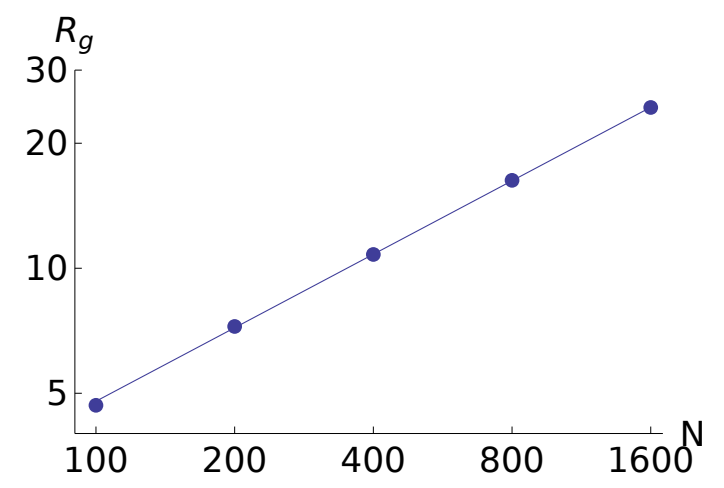

FIG. S3. $R_{g}$ as function of $N$ in $\log -\log$ scale. Symbols represent average radius of gyration of the sample The line is best fit to three largest size $N=400,800$ and 1600 and has exponent $\nu=0.587$. 


\section{MINIMAL SURFACE ON SPACE-FILLING CURVE}

We spanned minimal surfaces on a Moore curve (see Fig. S2 for an example) which is a closed version of the Hilbert curve. For Moore curve of lengths $N=64,512$ and 4096 we found the areas of the minimal surfaces to be 25.44, 218.79 and 1833.62 respectively, which corresponds to the same scaling as for the long rings from the melt $A \sim N^{x}$ with $x=1.03$ being the best fit. The contact exponent of the Hilbert curve is $\gamma=4 / 3$ as measured e.g. in $[6]$.

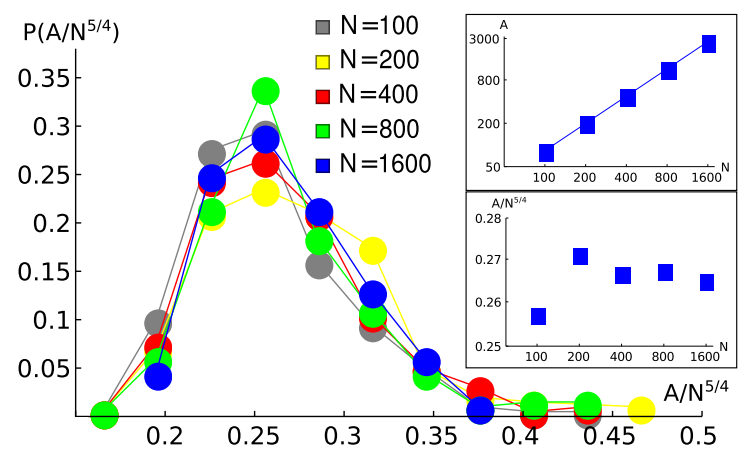

FIG. S4. Histogram of area of minimal surfaces for various lengths of swollen rings $N$ normalized by $N^{x_{S A W}}$ with $x_{S A W}=1.25$. The value of the exponent $x_{S A W}$ is the best fit of mean area $A$ (in units of bond length) versus $N$ (top inset), error bars are the same or smaller than the symbol size. Bottom inset: Mean area in units of bond length normalized by $N^{1.25}$

\section{FREELY FLUCTUATING UNKNOTTED RING CONFORMATIONS}

Unknotted ring conformations were prepared on a lattice by the BFACF algorithm $[7,8]$ that is known to be ergodic in any given knot class [9]. The BFACF algorithm is grand-canonical and we take the equilibrium probability of a ring conformation to be proportional only to the number of conformations of rings of a given length. This naturally drives the ring conformations to larger lengths, hence to control the ring length we employed the strategy used in [10]. We introduced Hamiltonian $H\left(N, N_{0}\right)$

$$
H\left(N, N_{0}\right)=\left\{\begin{array}{cl}
0 & \text { if } N \leq N_{0} \\
\left(N-N_{0}\right)^{2} & \text { if } N>N_{0}
\end{array} .\right.
$$

This produces almost uniform distribution of ring lengths $N$ around $N_{0}$.

The simulation starts from the smallest possible ring of $N=4$ and for each $N_{0}$ from $[100,200,400,800,1600]$ the integrated autocorrelation time $\tau_{\text {int }}$ of gyration radius $R_{g}$ was computed as in $[11,12]$. The conformations reached $N=N_{0}$, but were sampled only after $100 \tau_{\text {int }}$ after the start of the simulation $\left(30 \tau_{\text {int }}\right.$ for $\left.N_{0}=1600\right)$

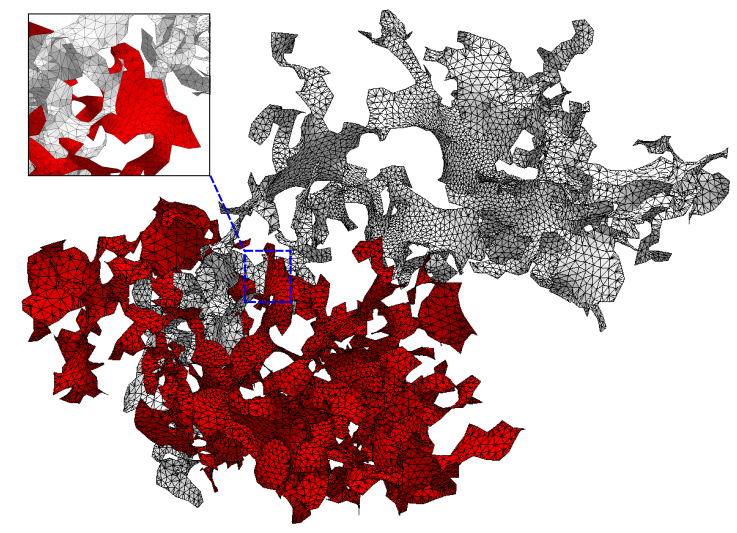

FIG. S5. Example of a ring threading of rings with $N=1600$. The ring with white surface crosses (top) the minimal surface of the ring with red surface (bottom). Inset: Close view of the threading ring. In this example $n_{p}=2$. For details please zoom into the electronic version of this figure.

which was found to be enough to reach equilibrium gyration radius. Then conformations with $N=N_{0}$ were sampled every $10 \tau_{\text {int }}$. To check the proper sampling we measured the mean square displacement of the center of mass of the ring and found it to be around $4 R_{g}^{2}$ in time $10 \tau_{\text {int }}$. We further analyzed scaling of the gyration radius of the conformations with length (Fig. S3) and found $R_{g} \sim N^{0.587}$ which is in agreement with the SAW exponent $\nu_{S A W}=0.588$.

The minimal surfaces for the freely fluctuating rings were obtained with the same annealing protocols as described above. Let us note that even though the monomers of these simulated rings were fixed to a lattice, their minimal surfaces were off-lattice. The normalized histogram of area of minimal surface in Fig. S4 shows good collapse for all ring lengths giving the exponent $x_{S A W}=1.25 \pm 0.07$. Although small deviations for smaller rings occur which can be a relict of their lattice nature.

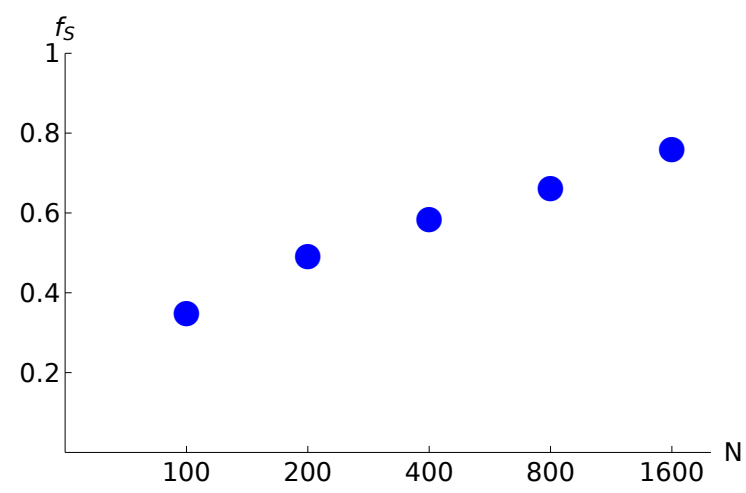

FIG. S6. Fraction of symmetric penetrations in log-linear scale as function of $N$. This is number of ring pairs in which ring $A$ penetrates ring $B$ and vice versa, divided by number of pairs of rings that are involved in any kind of penetration. 


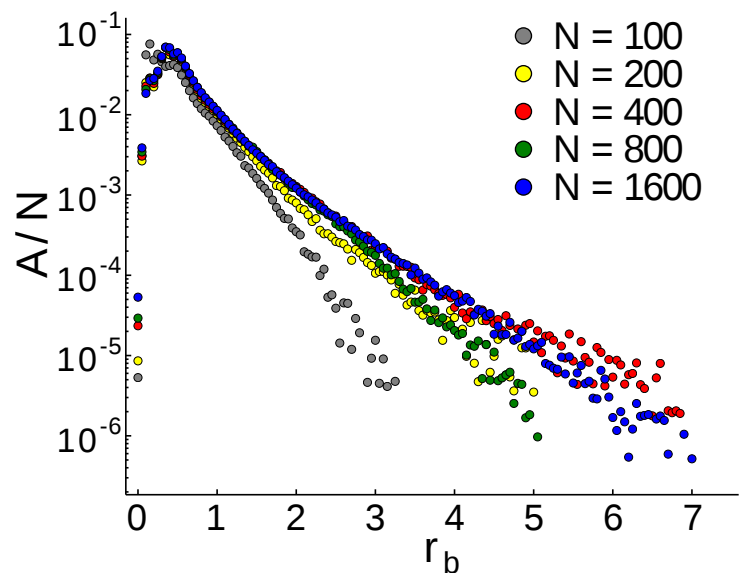

FIG. S7. Distribution of area of minimal surface per bond for the rings from melt with respect to the smallest distance $r_{b}$ from a boundary monomer.

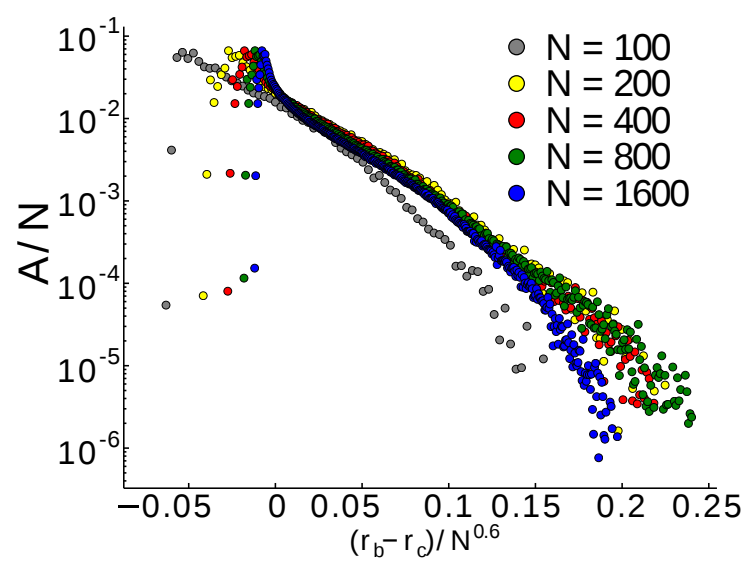

FIG. S8. Distribution of area of minimal surface per bond for the freely fluctuating with respect to the smallest distance $r_{b}$ from a boundary monomer. Offset $r_{c}=1.0$ was used due to lattice nature of the rings. The distance from the boundary was normalized by $N^{0.6}$.

\section{FRACTION OF MINIMAL SURFACE AREA CLOSE TO THE BOUNDARY}

Similarly to the analysis of minimal surface by M. Lang [13], we plot the area of the minimal surface per monomer as function of its distance $r_{b}$ from the nearest monomer for the rings in melt (Fig. S7) and freely fluctuating rings (Fig. S8).

In the case of freely fluctuating rings we collapsed the exponential tail by normalization factor $N^{0.6}$, consistent with finding of M. Lang. The distributions of the long rings from melt exhibit good collapse at intermediate distances $1<r_{b}<3$ without any normalization, which contrasts with the results of Lang, who used normalization by $N^{1 / 3}$. We address this difference to the fact that our rings and well minimized surfaces are off lattice and we find that almost whole area lies at the boundary. To see what is the contribution of the area located close to the boundary to the total area for different ring lengths, we plot separately the area per segment of triangles that have $r_{b}<1$ and those that are further away from the boundary. The result is in Fig. S9. This clearly demonstrates, that for the rings in the melt, the substantial part of the minimal surface is at the boundary which underlines the tree-like interpretation of the rings' conformation.

For the longest freely fluctuating rings, the area away from boundary dominates and scales with an effective exponent around 1.5 with $N$ i.e. $A\left(r_{b}>1\right) \sim N^{1.5}$. This is similar to the finding in [13].

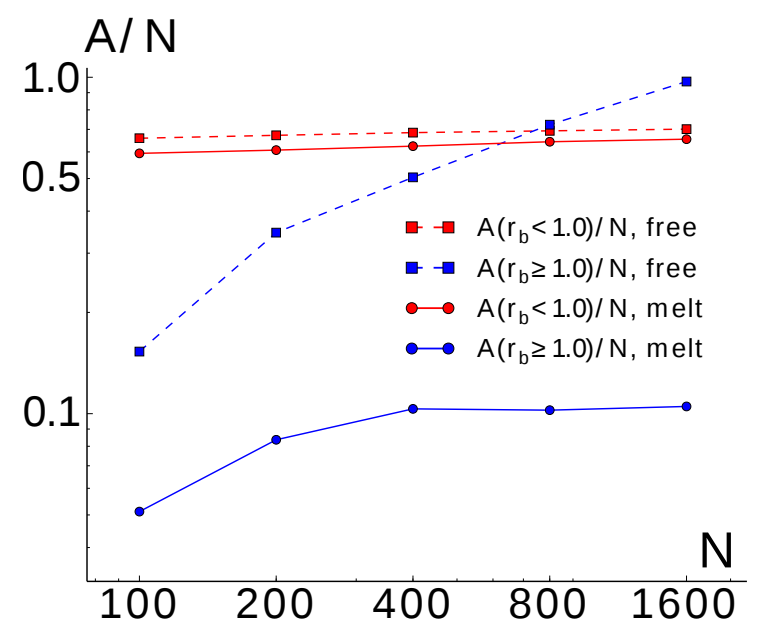

FIG. S9. Area of minimal surface per bond that lies close to the boundary $\left(r_{b}<1\right)$ and further away from the boundary $\left(r_{b}>1\right)$, for rings from melt (solid line) and freely fluctuating rings (dashed) in log scale. The best power law fit of $A\left(r_{b}>1\right)$ of the freely fluctuating rings with $N \geq 200$ is $A\left(r_{b}>1\right) \sim$ $N^{1.5}$

\section{RING THREADING EXAMPLE}

In figure S5 we show an example of ring threading.

Unfortunately we can not measure the area related to a single penetration event as we see cases of more penetrations being in mutual proximity on the surface (as in Fig. S5). It would be interesting to assess the uniformity of penetration distribution on the surface, e.g. by quantifying their mutual surface (geodesic) distances. However, we believe that physically more relevant quantities of the problem are related to the penetration length which obstructs the ring relaxation. Moreover we think that our analysis can provide some insight even without such a challenging exercise: On small scales the threading length distribution $p\left(L_{t}\right)$ reflects the random walk statistics hitting an infinite planar surface that locally approximates the minimal surface. Therefore it is reasonable to expect that the distribution of the relative position of the two penetrations on the surface would be gaussian with mean distance on the surface proportional 

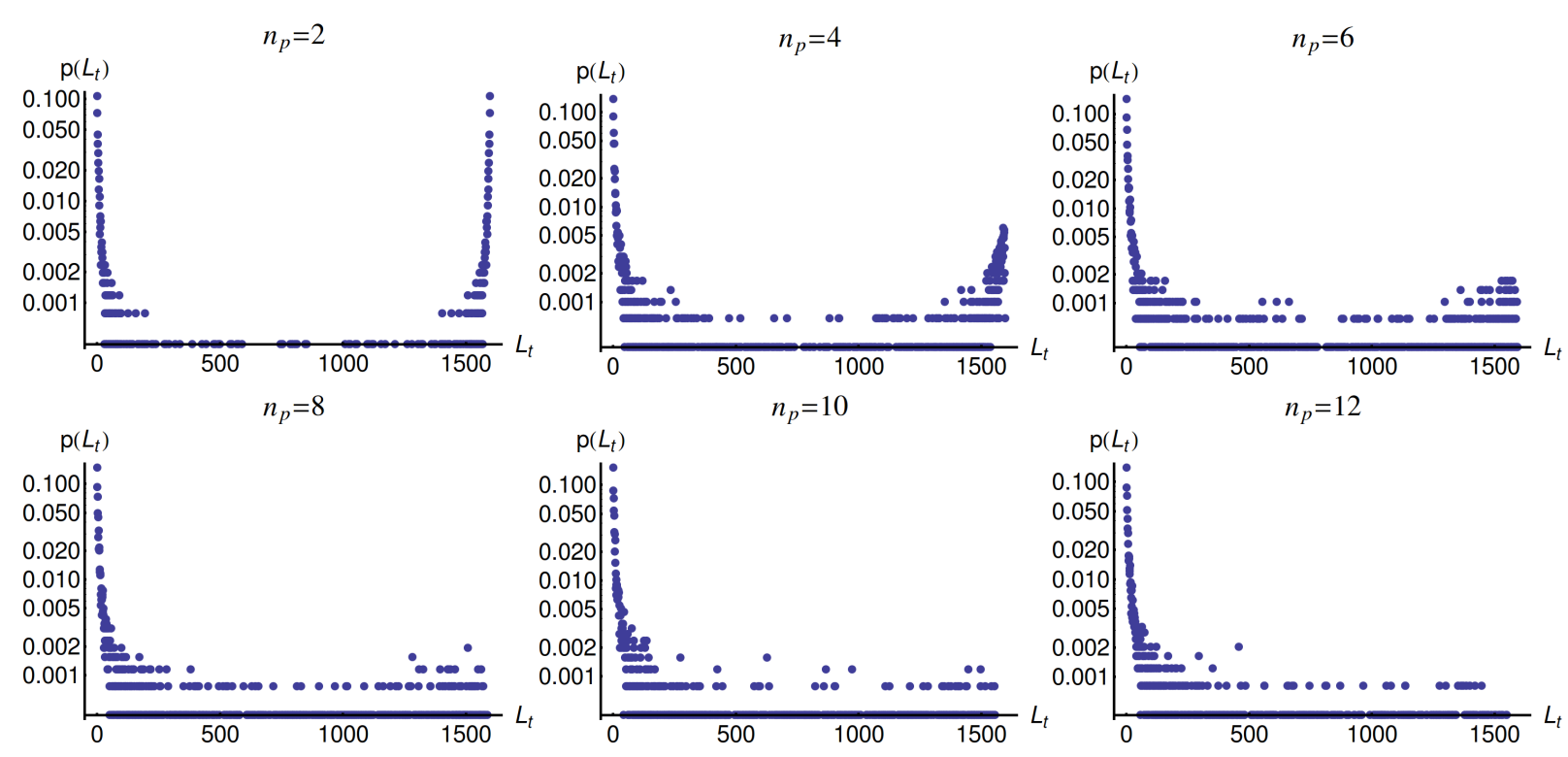

FIG. S10. Distributions of threading lengths only for rings with given $n_{p}$. The increase for large $L_{t}$ is present up to $n_{p}=8$.

to $L_{t}^{1 / 2}$, which are the predictions of such a model. On longer scales, however, both, the flat surface approximation and the random walk model fail, but at these scales there are few penetrations.

\section{FRACTION OF SYMMETRIC PENETRATIONS}

When ring $A$ penetrates ring $B$ and ring $B$ penetrates $\operatorname{ring} A$ we call this a symmetric penetration. In figure $\mathrm{S} 6$ we plot the fraction of the symmetric penetrations $f_{S}$ as function of $N$. With increasing $N$ the fraction of symmetric penetrations grows. This is expected because the number of neighbors of a given ring saturates due to their compact conformation, while the number of monomers of a ring in proximity of monomers of neighboring ring grows with $N$ as $N^{\beta \simeq 0.95}$ [14], which present more opportunities for mutual threading. The latter property is manifested in our data by the exponential distribution of number of penetrations $n_{p}$ with increasing mean as function of $N$ (Fig. 2). Therefore, in the limit of very long rings we expect $f_{S}$ to go to unity.

\section{THREADING LENGTH DISTRIBUTION}

Distribution of threading length for rings with given number of penetrations $n_{p}$ are shown in figure S10 up to ten penetrations.

A naive analytical model of the complete (i.e. for all $n_{p} \geq 2$ ) distribution $p\left(L_{t}\right)$ can be constructed in the following way. Let's consider at first a ring divided into $n_{p}$ segments and assume for simplicity that length of each segment is distributed independently uniformly ran-

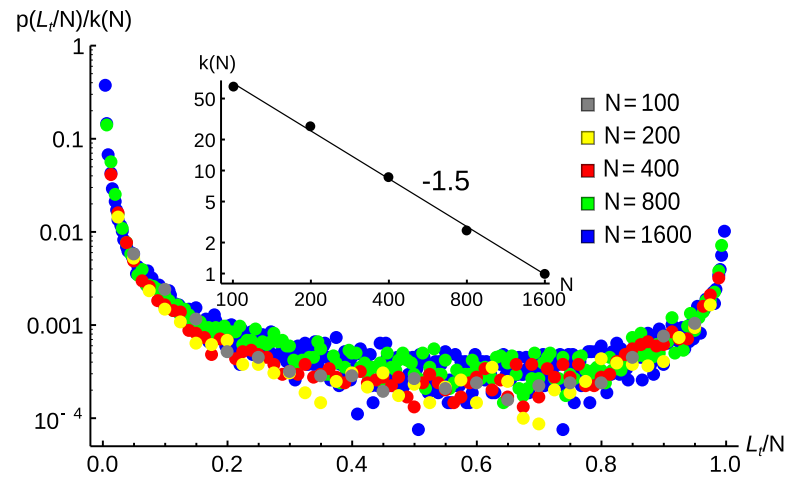

FIG. S11. (Color online) Collapse of distributions of the threading lengths $p\left(L_{t}\right)$ normalized by a length dependent factor $k(N)$, and $L_{t}$ is normalized by $N$. Inset: The factor $k(N)$ dependence on $N$ shows roughly power law dependence $N^{-1.5}$.

domly on $[0, N]$ with the obvious constraint that the total length must be that of the whole ring $N$. The distribution of threading lengths for $n_{p}$ penetrations is therefore $p_{n_{p}}(y)=\left(n_{p}-1\right)(1-y)^{n_{p}-2}$ with $y=L_{t} / N$. This is clear from the fact that the cumulative distribution function of this quantity $P_{n_{p}}(y)$ is the probability that at least one segment is of length shorter than $y$ or in other words 1 minus probability that all of the segments are longer than $y$. Therefore $P_{n_{p}}(y)=1-(1-y)^{n_{p}-1}$, because the $n_{p}-1$ segments define the length of the $n_{p}$-th segment by the total length constraint. Already the result for $p_{n_{p}}(y)$ is not consistent with our numerical findings in fig.S10 as it is nondecreasing only for $n_{p}=2$. The increase of the tail of the threading length distribution can not be recovered even if we calculate the complete $p\left(L_{t}\right)$ in the present model. As we found out, the number 
of penetrations $n_{p}$ of a ring is exponentially distributed with probability $\pi\left(n_{p}\right)=\left(1-w^{2}\right) w^{n_{p}-2}$ for some $w<1$. The normalization takes into account that the rings with $n_{p}=0$ are not considered in the statistics and only even number of penetrations is possible due to topology constraint of nonconcatenation. Therefore the distribution $p\left(L_{t} / N\right)=p(y)$ is

$$
p(y)=\sum_{n_{p}}^{\infty} \pi\left(n_{p}\right) p_{n_{p}}(y)=\frac{\left(1-w^{2}\right)\left(1+(w(1-y))^{2}\right)}{\left(1-(w(1-y))^{2}\right)^{2}},
$$

which is decreasing. Similarly, monotonous decay is seen even with exponential distribution for each segment length. These observations suggest that to explain the increase in $p\left(L_{t}\right)$ some form of dependence or correlation between subsequent surface penetrations seems necessary.

As mentioned in the main text, we can collapse most of threading length distributions for different $N$ by normalizing $L_{t}$ by $N$, and $p$ by a length dependent factor $k(N)$ (Fig. S11). The factors $k(N)$ are obtained as a result of least square minimization of the difference between the distributions. The factors exhibit roughly scaling $k(N) \sim N^{-1.5}$. Similarly, one can collapse the curves, by normalization of $p$ by its minimal value $\min (p)$, which exhibit similar scaling, but the collapse is slightly worse (not shown).

\section{SEPARATION LENGTH DISTRIBUTION}

In figure S12 we show the distribution of the separation length calculated from the threading length data as explained in the main text and the inset of the figure. The effective slope is in the range of -1.1 and -1.5 depending on the lengthscale, which is somewhat higher as in the case of the threading length, but we can not draw a more definite conclusion. Interesting is however the clear change of the distribution if only a subclass of neighbors that remain together is taken into account as was discussed in the main text.

The $L_{s e p}$ is composed on average of $n_{p} / 2$ protruding segments (two penetrations per segment that belongs to $\left.L_{s e p}\right)$. If an average protruding segment is significantly longer than $N_{e}$ i.t presents a topological obstacle for the other rings and other opportunities for threading. If every ring has many neighbors with such long protrusions, the threading is expected to hinder the dynamics. Therefore it is interesting to look at how many threaded neighbors a ring has as function of the separation length.

In Fig. S13 we plot the cumulative distribution of number of threaded neighbors $n_{t}\left(L_{\text {sep }} /\left(n_{p} / 2\right)\right)$ of a threading ring. The length $L_{s e p} /\left(n_{p} / 2\right)$ reflects the separation length per protruding segment and in the figure it is plotted in units of the entanglement length. This means that $n_{t}(x)$ gives the number of neighbors that are threaded by a ring with separation length longer, or equal to $x n_{p} / 2$,

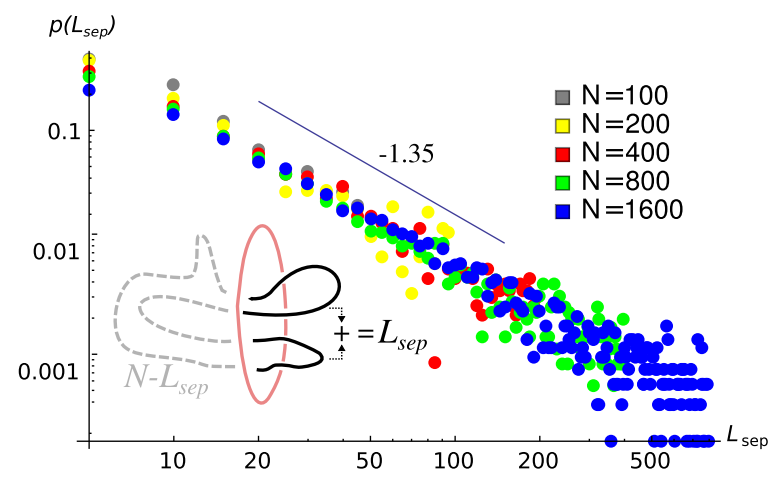

FIG. S12. Distribution of separation length for different $N$ in $\log$-log scale. The effective slope -1.35 is shown as a guide to the eye; Inset: Example of separation length as the total length of two black threading length segments, corresponding to $L_{t 1}$ and $L_{t 3}$ in figure 3 . The remaining part (dashed gray) has total length $N-L_{\text {sep }}$.

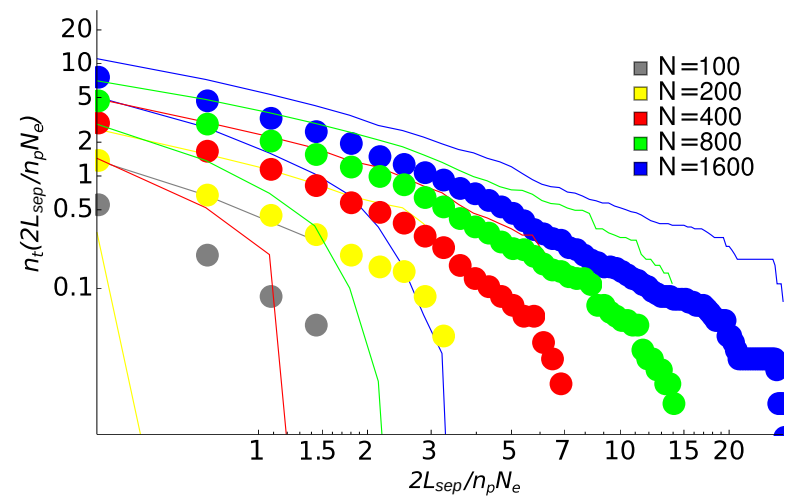

FIG. S13. Cumulative distribution of average number of threaded neighbors of a ring as function of the average length of one protrusion in units of $N_{e}$ in log-log scale for all ring lengths. Symbols represent mean and thin lines are ensemble standard deviation error bars. See text for more explanations and examples.

and specifically $n_{t}(0)=n_{t}$ is the number of threaded (by any protrusion length) neighbors of a ring. We found (Fig. S13) that although there are many threaded neighbors, the decay of the distribution is pretty sharp: for example every ring of $N=1600$ has about one neighbor that is threaded by a protruding segment of length at least $3 N_{e}$ i.e. $n(3)=1$ for $N=1600$. While only about every other ring has a neighbor that is threaded by a segment of length at least $5 N_{e}$ i.e. $n(5) \simeq 0.5$ for $N=1600$. This may explain the success of the annealed tree model which neglects threadings. To understand this better one will have to work out perturbative corrections to the annealed tree model due to threadings; we leave this as a task for the future.

We see that the number of neighbors with a given penetration length is increasing as function of $N$, because, as already mentioned, even the longest rings have not yet reached the asymptotic regime. To see how close we are 


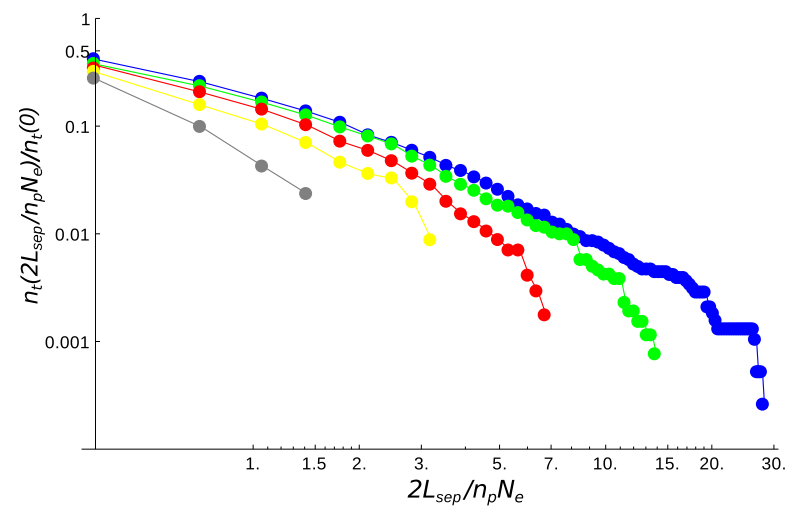

FIG. S14. Cumulative distribution of average number of threaded neighbors rescaled by the total number of threaded neighbors $n_{t}(0)$ as function of the average length of one protrusion in units of $N_{e}$ in log-log scale for all ring lengths.

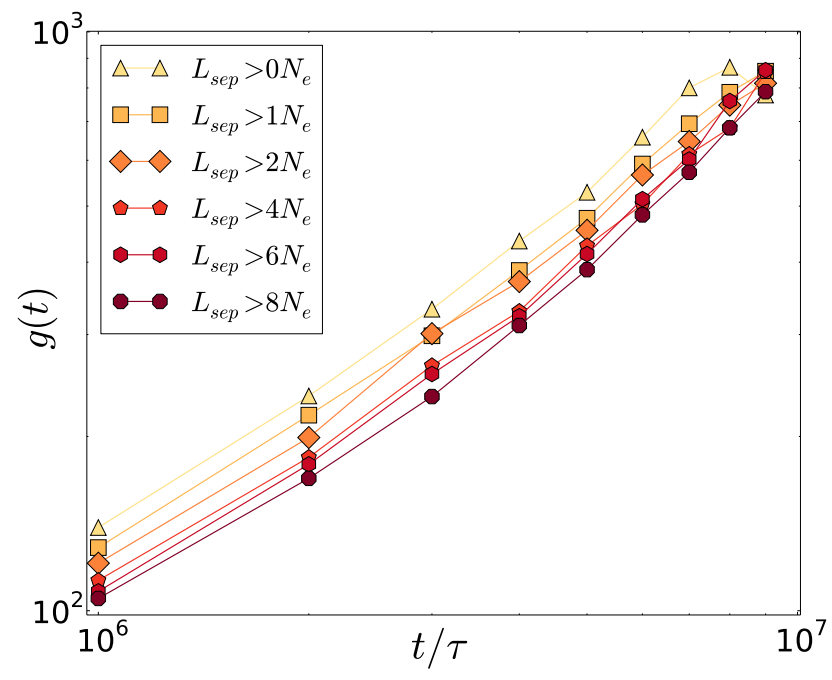

FIG. S15. Relative ring-ring mean square displacement as function of time for ring pairs ensembles defined by their minimal separation length. Each ensemble was resided to the size of the ensemble with $L_{\text {sep }}>8 N_{e}$, by re-sampling. with our longest rings to the asymptotic regime, in figure Fig. S14 we rescaled the $n_{t}\left(L_{s e p} /\left(n_{p} / 2\right)\right)$ by $n_{t}(0)$. We see that for the $N=800$ and $N=1600$ the difference quite small, therefore although there are many penetrations that are long it is not clear if even longer rings would make sufficiently many severely threaded neighbors to reach the topological glass regime.

\section{RELATIVE RING-RING MEAN SQUARE DISPLACEMENT: STATISTICS DETAILS}

The apparent decreasing relative mean square displacement $g$ as function of $L_{\text {sep }}$ could be caused, in principle, by smaller ensemble size for larger $L_{\text {sep }}$ and therefore undersampling rare ring pairs with large $g$. In the figure S15 we show that this is not the case, by normalizing ensemble size for each data point to the smallest ensemble for that given time (i.e. each ensemble was re-sampled to the size of the ensemble with $L_{s e p}>8 N_{e}$ at given time). Note that in this figure the ensemble is defined only by the lower bound of separation length, while in the main text an additional upper bound was used. The decreasing character of $g$ with $L_{s e p}$ is still clear, especially for early times. At later times we observe the rings have not yet fully recovered the delay.

There are still many open routes to explore. In this work, we were trying to depict some of these (minimal surface area scaling, ...) while at the same time keeping in view our main goals: $(i)$ understanding the seeming conflict between the success of the tree model and observation of the ring threading and (ii) analyzing the threading in order to understand implications for the ring dynamics.
[1] Courant, R. Soap film experiments with minimal surfaces. Am. Math. Monthly 47, pp. 167-174 (1940).

[2] Goldstein, R. E., Moffatt, H. K., Pesci, A. I. \& Ricca, R. L. Soap-film Möbius strip changes topology with a twist singularity. Proc. Natl. Acad. Sci. U.S.A. 107, 21979-21984 (2010).

[3] Goldstein, R. E., McTavish, J., Moffatt, H. K. \& Pesci, A. I. Boundary singularities produced by the motion of soap films. Proc. Natl. Acad. Sci. U.S.A. 111, 8339-8344 (2014).

[4] Brakke, K. Surface evolver program version 2.70. http://www.susqu.edu/brakke/evolver/evolver.html (1989-2013). Accessed: 2015-03-03.

[5] Scherk, H. F. Bemerkungen über die kleinste Fläche innerhalb gegebener Grenzen. J. reine angew. Math. 13, 185-208 (1835).
[6] Smrek, J. \& Grosberg, A. Y. A novel family of spacefilling curves in their relation to chromosome conformation in eukaryotes. Physica A 392, 6375 - 6388 (2013).

[7] Berg, B. \& Foerster, D. Random paths and random surfaces on a digital computer. Phys. Lett. B 106, $323-$ 326 (1981).

[8] Aragão de Carvalho, C., Caracciolo, S. \& Fröhlich, J. Polymers and $\mathrm{g}|\varphi|^{4}$ theory in four dimensions. Nucl. Phys. B 215, 209 - 248 (1983).

[9] van Rensburg, E. J. J. \& Whittington, S. G. The BFACF algorithm and knotted polygons. J. Phys. A: Math. Gen. 24, 5553 (1991).

[10] Mansfield, M. L. \& Douglas, J. F. Properties of knotted ring polymers. i. equilibrium dimensions. J. Chem. Phys. 133 (2010).

[11] Madras, N. \& Sokal, A. The pivot algorithm: A highly 
efficient Monte Carlo method for the self-avoiding walk. J. Stat. Phys. 50, 109-186 (1988).

[12] Caracciolo, S., Pelissetto, A. \& Sokal, A. Dynamic critical exponent of the BFACF algorithm for self-avoiding walks. J. Stat. Phys. 63, 857-865 (1991).

[13] Lang, M. Ring conformations in bidisperse blends of ring polymers. Macromolecules 46, 1158-1166 (2013).

[14] Halverson, J. D., Lee, W. B., Grest, G. S., Grosberg, A. Y. \& Kremer, K. Molecular dynamics simulation study of nonconcatenated ring polymers in a melt. i. statics. J. Chem. Phys. 134, 204904 (2011). 